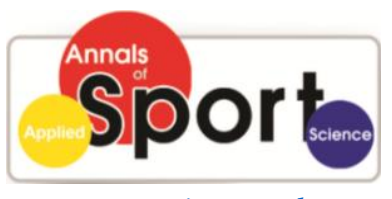

www.aassjournal.com

ISSN (Online): 2322 - 4479

ISSN (Print): 2476-4981

Editorial

WwW.AESAsport.com

Received: 03/01/2017

Accepted: 09/04/2017

\title{
Exercise Training, Neuregulin 4 and Obesity
}

\author{
${ }^{1}$ Seyed Morteza Tayebi, ${ }^{2}$ Abbas Ghanbari-Niaki*, ${ }^{2}$ Ayoub Saeidi, ${ }^{3}$ Anthony C. Hackney \\ ${ }^{1}$ Core Research of Health Physiology and Physical Activity, Department of Exercise Physiology, Faculty of Sport \\ Science, Allameh Tabataba'i University, Tehran, Iran. ${ }^{2}$ Exercise Biochemistry Division, Faculty of Sport Sciences, \\ University of Mazandaran, Babolsar, Iran. ${ }^{3}$ Department of Exercise \& Sport Science, University of North Carolina, \\ Chapel Hill, NC, United States.
}

\section{DEAR EDITOR}

Obesity is the accumulation of excess fat tissue on the body due to an imbalance between food intake and energy expenditure (1). Accumulations of excess fat are strongly related to the development of different metabolic risk factors such as type 2 diabetes mellitus, hypertension, dyslipidemia and chronic inflammation which result in the development of debilitating cardiovascular diseases (2). However, the precise molecular mechanism of the pathophysiological alterations with obesity are not fully recognized, but substantial research states that abnormal secretion of adipokines and lipid metabolism by adipocyte are involved in the phenomenon $(2,3)$. In recent years, attitudes have been changed about adipose tissue as being an inert tissue and nowadays it is considered as a vital endocrine tissue that secretes several proteins and adipokines that play an important physiological role in the control of energy homeostasis $(2,3)$.

Neuregulin $4(\mathrm{Nrg} 4)$ is one of this adipokines secreted by adipose tissue (primarily brown adipose tissue [BAT]) which especially targets the liver (3). Neuregulin is a member of the epidermal growth factors (EGF) family of receptor kinases, which was originally identified as the product of the transforming gene derived from chemically induced rat neuroblastoms. A variety of different protein isoforms are produced from the single Neuregulin gene; such as, the four distinct vertebrate gene encoded Neuregulin named NRG1, NRG2, NRG3, and NRG4 $(4,5)$. Nrg4 aids in maintaining energy balance via its ability to limit hepatic lipogenesis. Also it has been observed that Nrg4 is expressed higher when BAT is exposure to cold and epinephrine. Nrg4 transfers its signals via activating ErbB receptors especially the type ErbB4 and ErbB3 receptors, for this reason the liver is a key target tissue for Nrg4. In order to investigate the energy balance role of $\mathrm{Nrg} 4$, Researchers have studied Nrg4 knockout rats with a normal and high-fat diet.. With a high-fat diet Nrg4 knockout rats have a greater increase in weight and body fat, and showed reduction in fat free mass, as well as having higher plasma triglyceride and fasting blood glucose and insulin levels. On the other hand glucose tolerance or insulin sensitivity test showed that the lack of Nrg4 exacerbated glucose intolerance and insulin resistance in rats with a high-fat diet. It has been reported $\mathrm{Nrg} 4$ knockout rats have higher triglyceride content and increased plasma concentrations of alanine aminotransferase (3).

*. Corresponding Author:

Abbass Ghanbari-Niaki

E-mail: ghanbara@umz.ac.ir 
Interestingly, a large study of gene expression in the liver of these animals indicated that the absence of $\mathrm{Nrg} 4$ was associated with induction of mRNAs of many actors of the lipogenic pathway (fatty acid synthase, acetyl-CoA carboxylase, malic enzyme, stearoyl-CoA desaturase-1. . .) (3). This was secondary to a major increase in the transcriptional factor controlling lipogenesis, SREBP1. By contrast, in $\mathrm{Nrg} 4$ knockout, the mRNA expression of genes involved in fatty acid oxidation, gluconeogenesis, or mitochondrial oxidative phosphorylation remained unmodified. The absence of Nrg4 therefore results in abnormal induction of hepatic lipogenesis and predisposes the animals to high fat diet-induced fatty liver. In order to explore a direct action of $\mathrm{Nrg} 4$ on hepatic lipogenesis, primary cultures of mouse hepatocytes expressing ErbB4 receptor were used (3). Under these conditions, lipogenesis induced by an agonist of the LXR nuclear receptor was markedly inhibited in the presence of $\mathrm{Nrg} 4$, which behaves as a potent antilipogenic effector, causing trans-repression of gene encoding LXR (3).

However, in rats with high-fat diet or genetically obese $^{\mathbf{o b} / \mathbf{o b}}$ and ${ }^{\mathbf{d b} / \mathbf{d b}}, \mathrm{Nrg} 4$ expression significantly decreases in epididymal white fat and BAT. In a large group of people with a high body mass index (BMI), Nrg4 mRNA expression in subcutaneous fatty tissue is negatively correlated with BMI and liver fat content (3). In addition, after adjusting subjects based on BMI, Nrg4 mRNA expression levels in patients with impaired glucose tolerance or type 2 diabetes was lower compared to subjects with normal glucose tolerance. This observational data in humans is associated with liver phenotype of rats lacking Nrg4 that shows insufficient expression of $\mathrm{Nrg} 4$ in fatty tissue can be involved in the pathogenesis of nonalcoholic fatty liver disease. Accordingly, it may be anticipated that overexpression of $\mathrm{Nrg} 4$ can prevent (or lessen) metabolic disorders associated with obesity (3). Thus, the discovery of this new adipokine with strong hepatic antilipogenic activity and beneficial effects on glucose and lipid metabolism, suggests new therapeutic opportunities in the field of nonalcoholic steatohepatitis.

On the other hand, among factors to prevent obesity and related diseases such as diabetes and fatty liver, exercise training is a well-accepted as a viable option (6). However, due to the effect of exercise training on fat tissue and obesity, and reduction in adipokines associated with insulin resistance such as leptin, retinol-binding protein 4 (7), resistin and others, as well as increases in adiponectin levels with exercise training, study of the effects of exercise training on $\mathrm{Nrg} 4$ are warranted and could create a broad new approach in relation to the prevention of fatty liver, obesity and insulin resistance treatment.

\section{REFERENCES}

1. Ortega L, Riestra P, Navarro P, Gavela-Pérez T, Soriano-Guillén L, Garcés C. Resistin levels are related to fat mass, but not to body mass index in children. Peptides. 2013;49:49-52.

2. Fasshauer M, Blüher M. Adipokines in health and disease. Trends in Pharmacological Sciences. 2015;36(7):46170.

3. Fève B, Bastard C, Fellahi S, Bastard J-P, Capeau J. New adipokines. Annales d'Endocrinologie. 2016;77(1):4956.

4. Arslan KS, Akpunar F, Ulucan K. Can Neurogulin 1 be an Important Biomarker for Creativity in Sports? Annals of Applied Sport Science. 2016;4(1):1-2.

5. Ghanbari-Niaki A. Neuregulins Response to Exercise: a Mini Review. Annals of Applied Sport Science. 2016;4(1):3-7.

6. Ghanbari-Niaki A, Saeidi A, Aliakbari-Beydokhti M, Ardeshiri S, Kolahdouzi S, Chaichi MJ, et al. Effects of Circuit Resistance Training with Crocus Sativus (Saffron) Supplementation on Plasma Viscosity and Fibrinogen. Annals of Applied Sport Science. 2015;3(2):1-10.

7. Tayebi SM, Hasannezhad P, Saeidi A, Fadaei MR. Intense Circuit Resistance Training along with Zataria multiflora Supplementation Reduced Plasma Retinol Binding Protein-4 and Tumor Necrosis Factor- $\alpha$ in Postmenopausal Females. Jundishapur J Nat Pharm Prod.In press(In press):e38578. 